\title{
Emotional distress in haemophilia: Factors associated with the presence of anxiety and depression symptoms among adults
}

\author{
P. R. Pinto ${ }^{1,2 *}$ (D) | A. C. Paredes ${ }^{1,2 *}$ | P. Moreira ${ }^{1,2}$ | S. Fernandes ${ }^{3}$ | M. Lopes ${ }^{3}$ | \\ M. Carvalho ${ }^{3}$ | A. Almeida ${ }^{1,2}$
}

${ }^{1}$ Life and Health Sciences Research Institute (ICVS), School of Medicine, University of Minho, Braga, Portugal

${ }^{2}$ ICVS/3B's-PT Government Associate Laboratory, Braga/Guimarães, Portugal

${ }^{3}$ Centre of Hemophilia, Department of Transfusion Medicine and Blood Bank, Centro Hospitalar São João, Porto, Portugal

\section{Correspondence}

Patrícia Ribeiro Pinto, Life and Health Sciences Research Institute, School of Medicine, University of Minho, Braga, Portugal.

Email: patipinto@med.uminho.pt

Funding information

Novo Nordisk HERO Research, Grant/Award Number: 2015; Portuguese Foundation for Science and Technology, Grant/Award Number: SFRH/BPD/103529/2014
Introduction: Haemophilia is related to several clinical and psychosocial challenges that have been associated with increased emotional distress. These may impact on disease adjustment and health outcomes, reinforcing the attention given to psychosocial health of people with haemophilia (PWH), in the scope of optimal comprehensive care.

Aim: To identify potentially modifiable factors associated with the presence of anxiety and depression symptoms among adult PWH.

Methods: This was a cross-sectional observational study examining sociodemographic, clinical and psychosocial variables among 102 patients with haemophilia A or B who participated on a mail survey of haemophilia in Portugal.

Results: People with haemophilia revealing higher anxiety and depression symptoms were more likely to have had, in the previous year, more urgent hospital visits due to haemophilia, more bleeding episodes, more affected joints and pain, as well as worst levels of perceived functionality and quality of life. After controlling for demographic (age and education) and clinical (haemophilia severity and joint deterioration) variables in multivariate hierarchical logistic regression analyses, professional status $(O R=4.646, P=.004 ; O R=3.333, P=.029)$ and pain interference $(O R=1.397$, $P=.011 ; \mathrm{OR}=.1 .347, P=.037)$ were significantly associated with both anxiety and depression symptoms. Additionally, physical activity $(O R=0.302, P=.024)$ and the perception of consequences underlying haemophilia $(O R=1.600, P=.012)$ also emerged as key factors significantly associated with depression symptoms.

Conclusion: Current findings increased knowledge on factors associated with anxiety and depression among PWH. These highlight potential intervention targets, which are amenable to change through evidence-based tailored interventions aiming to decrease emotional distress, promote well-being and improving haemophiliarelated health outcomes among these patients.

\section{KEYWORDS}

anxiety, depression, haemophilia, illness perceptions, pain, psychological intervention 


\section{1 | INTRODUCTION}

Haemophilia is a rare genetic disorder associated with a pattern of spontaneous bleeding that is the hallmark of the disease. ${ }^{1}$ Factor replacement therapy remains the mainstay of treatment, aiming to increase coagulation factor levels, in order to prevent or to stop bleeds. $^{2}$

Despite much improvement in treatment, people with haemophilia (PWH) still face many important clinical challenges, such as demanding treatment regimens, severe acute pain related to haemarthrosis, debilitating haemophilic arthropathy or inhibitor development, ${ }^{2,3}$ which are associated with increased emotional distress. ${ }^{3-5}$

Beyond clinical manifestations, $\mathrm{PWH}$ also cope with challenging psychosocial issues, ${ }^{6}$ such as uncertainty, social restriction and unemployment, which have also been associated with higher emotional disturbance. ${ }^{4,5,7}$ As such, current guidelines for optimal haemophilia care establish psychosocial health promotion as a priority, along with the prevention of bleeds and management of complications. ${ }^{1}$ This is particularly relevant considering that medical treatments alone may not be sufficient to address some of the challenges faced by PWH. ${ }^{6}$ Moreover, high levels of distress have a detrimental impact on adherence to factor replacement therapy and disease management, potentially affecting haemophilia-related health outcomes. ${ }^{8,9}$ Given these associations, and regardless of whether emotional distress is a cause or a consequence of disease and treatment issues, a promising strategy for improving haemophilia outcomes would therefore be the effective management of emotional distress experienced by patients. A thorough psychosocial assessment, including an appropriate anxiety and depression screening, is therefore warranted in haemophilia care, towards a more systemic approach and integrated care. ${ }^{8,10}$ Nevertheless, the screening of anxiety and depression in PWH has been either undifferentiated and overlapped or based on relatively unspecific evaluation of symptoms. This assessment often relies on general mental health dimensions assessed by quality of life questionnaires, ${ }^{7}$ which might hinder accurate diagnosis. Therefore, the use of specific standardized measures validated for anxiety/depression screening would be an essential step towards a more precise evaluation of distress levels among PWH. Concurrently, it is also crucial to identify modifiable factors that are associated with anxiety and depression, in order to inform the development of tailored treatments targeting these key outcomes. In this scope, the effectiveness of psychological interventions is well established, ${ }^{11,12}$ with some reports suggesting the usefulness and cost-effectiveness of these interventions in the improvement of coping strategies and emotional well-being among $\mathrm{PWH}^{13,14}$

This study aimed to investigate which potentially modifiable factors were associated with the presence of anxiety and depressive symptoms among adults with haemophilia. Ultimately, this contributes to uncover intervention targets that are amenable to change or to active management, and therefore suitable to inform the development of tailored psychological interventions for $\mathrm{PWH}$.

\section{2 | MATERIALS AND METHODS}

\section{1 | Design and participants}

This study is part of a large cross-sectional observational study conducted among Portuguese PWH registered in the Portuguese Haemophilia Association (APH). According to the World Federation of Haemophilia, there is a reported prevalence of $703 \mathrm{PWH}$ in Portugal (haemophilia A: 539; haemophilia B: 112; type unknown:52). ${ }^{15}$ Of these, $500 \mathrm{PWH}$ were reached by mail from October 2016 to May 2017 to complete a survey concerning sociodemographic, clinical and psychosocial characteristics of $\mathrm{PWH}$, which was returned by 146 participants (29.2\% return rate). More detailed information concerning survey procedure and patient recruitment can be found elsewhere. ${ }^{16}$ From the 106 adults returning the survey, 4 were excluded due to missing data on the anxiety or depression measures, leaving 102 participants in the final sample. For the purposes of current study, wherein the focus is on emotional distress, data concerning the assessments of anxiety and depression were retained to be analysed as the main outcome. Participants included in this study were males with haemophilia $A$ or B of any severity and age over 18 years old. Ethical approval was obtained from the Ethical Committee at University of Minho and the Portuguese Data Protection Agency, and written informed consent was obtained from all the participants. The study is registered at clinicaltrials.gov (NCT02870114).

\subsection{Data collection}

Data were collected using the Portuguese versions of the following questionnaires. The tools that had not yet been adapted to European Portuguese (A36 Hemofilia-QoL and Haemophilia Activities List) underwent a complete translation-back translation process followed by pilot testing, conducted by our team for the purposes of this investigation.

Socio-Demographic and Clinical Questionnaire: collects sociodemographic, clinical (eg bleeds and affected joints) and physical activity data. Participants were asked if they practiced some form of physical activity, either regularly or occasionally, and provided information on the frequency and types of activities.

Pain Questionnaire: Information regarding pain was evaluated with single-questions asking about the presence of pain in the previous year, if it lasted over 3 months or occurred more than once a week (yes/no format); and about pain duration (in months). Intensity was assessed through a 0-10 Numerical Rating Scale (NRS) $(0=$ no pain; 10 = worst imaginable pain) in six situations (eg bleeding episodes, using stairs, sitting or lying down). Interference score was based on the Brief Pain Inventory (BPI) interference subscale, ${ }^{17}$ according to 7 dimensions rated on an 0-10 NRS ( 0 = no interference; $10=$ completely interferes). An average total score is computed to reach intensity $(\alpha=0.88)$ and interference $(\alpha=0.91)$ global scores.

PROMIS ${ }^{\circledR}$ Depression and Anxiety short-forms ${ }^{18}$ : measure symptoms of anxiety $(\alpha=0.83)$ and depression $(\alpha=0.92)$ with 4 items each. Scores range from 4 to 20 , with higher scores indicating more 
severe symptoms. A score of 8 has been proposed as the cut-off for clinically relevant symptoms in both scales. ${ }^{19}$

Illness Perception Questionnaire-Revised (IPQ-R $)^{20}$ : assesses perceptions and beliefs about haemophilia through a psychometrically shortened 21 -item version, ${ }^{21}$ considering seven subscales: timeline acute/chronic ( $\alpha=0.63$ ), timeline cyclical $(\alpha=0.70$ ), consequences $(\alpha=0.76)$, personal control $(\alpha=0.52)$, treatment control $(\alpha=0.42)$, illness coherence $(\alpha=0.73)$ and emotional representation $(\alpha=0.87)$. The scores for each dimension range between 3 and 15, with higher values translating more threatening illness perceptions, and no global score is computed for the IPQ-R. Due to missing data in all subscales, missing data patterns were examined using the Little's MCAR test. ${ }^{22}$ Using this statistical procedure, it was observed that all incomplete information ( $<3 \%$ of the entries in the dataset) were "missing completely at random" (MCAR; $\chi_{(302)}^{2}=292.48, P=.642$ ). As such, a Multiple Imputation (MI) method was used to calculate the missing data, based on the Expectation Maximization (EM) algorithm, which is considered an unbiased strategy to impute missing data information. ${ }^{23}$

A36 Hemofilia-QoL ${ }^{24}$ : evaluates haemophilia-specific quality of life through 36 items. A percentile score can be computed (0-100 percentile) for the global scale $(\alpha=0.96)$, with higher scores translating better quality of life.

Haemophilia Activities List (HAL) ${ }^{25}$ : assesses patients' selfperceived functionality, namely the difficulty in performing activities due to haemophilia through 42 items ( $\alpha=0.98$ ), with global score ranging from 0 (worst functional status) to 100 (best functional status).

\subsection{Statistical analysis}

Data analysis was conducted using the IBM SPSS ${ }^{\circledR}$ version 24 software (Chicago, IL, USA) and the IBM SPSS ${ }^{\circledR}$ Amos version 24 for data imputation.

Descriptive statistics for continuous variables are presented as median and range, whereas categorical data are presented as numbers and percentages. Internal consistency of responses to the Portuguese versions of psychosocial questionnaires was assessed using Cronbach's alpha. ${ }^{26}$

Given the aim of this paper of uncovering factors associated with the presence of anxiety/depression symptoms, the two primary outcomes were assessed as dichotomous variables: presence (PROMIS $\geq 8$ ) or absence (PROMIS $<8$ ) of significant anxiety and depression symptoms. ${ }^{19}$ Distribution of predictive data differed significantly from normality assumptions. Thus, Mann-Whitney (for continuous variables) and chi-square tests or the Fisher exact test (for nominal variables) were performed to compare groups. To determine the meaningfulness of the differences (practical significance), since statistical significance ( $P$-value) is dependent on group size, the associated effect sizes were also computed. They were expressed as $r$ score for continuous variables and Pearson's phi $(\Phi)$ coefficient for nominal variables.

Finally, a set of predictive hierarchical multivariate logistic regression models were conducted to analyse the factors associated with the presence of anxiety and depression symptoms. The variables selected for inclusion in the models were those which better distinguished between either the anxiety or the depression groups in univariate analysis, both in terms of statistical significance $(P \leq .001)$ and simultaneously showing at least a medium effect size $(>0.30){ }^{27}$ In addition, all the comparisons were adjusted for relevant sociodemographic and clinical variables: age, education, haemophilia severity and number of affected joints.

Considering the potential shared variance among the independent factors, and due to the small sample size, four different submodels were considered for each distinctive outcome-anxiety and depression. The first model focused on the role of professional status, the second centred on physical activity practice, the third model explored the role of pain (intensity and interference) and, finally, the fourth model addressed illness perceptions (timeline cyclical, consequences and emotional representation related to haemophilia). Statistically significant results were considered for $P<.05$. Multicollinearity was analysed through the variance inflation factor value $(\mathrm{VIF}<2)$ and the tolerance coefficients $(>0.60)$ for each variable. $^{28}$

\section{3 | RESULTS}

\section{1 | Association of emotional distress with clinical and psychosocial outcomes}

In the present sample, significant (PROMIS $\geq 8$ ) anxiety and depression symptoms were found on 37 (36.3\%) and 28 (27.5\%) participants, respectively. Figure $1(\mathrm{~A}-\mathrm{F})$ illustrates the differences among participants with and without significant anxiety/depression symptoms on key haemophilia-related clinical and psychosocial outcomes.

\section{2 | Characteristics and differences between groups of patients with and without anxiety and depression}

Table 1 shows that, for both anxiety and depression, participants who revealed significant clinical symptoms (PROMIS $\geq 8$ ) were older than those without those symptoms (anxiety: $P=.018, r=.260$; depression: $P=.04, r=.204$ ) and were more often unemployed, retired or on medical leave (anxiety: $P<.001, \Phi=0.411$; depression: $P<.001, \Phi=0.357)$. Regarding physical activity, its practice was significantly associated with less symptoms of both anxiety $(P=.038$, $\Phi=0.206)$ and depression $(P=.001, \Phi=0.324)$. The groups did not differ significantly in any of the other socio-demographic measures.

Table 1 reveals that those presenting either anxiety or depression were more likely to report bleeds $(P=.019, \Phi=0.245 ; P=.006$, $\Phi=0.284$; respectively) and urgent hospital visits due to haemophilia in the previous year $(P=.005, \Phi=0.290 ; P=.012, \Phi=0.258$; respectively), also indicating a higher number of affected joints $(P<.001, r=.386 ; P<.001, r=.284$; respectively). Pain was more often reported among participants presenting significant anxious $(P=.004, \Phi=0.288)$ and depressive $(P=.001, \Phi=0.317)$ symptoms 

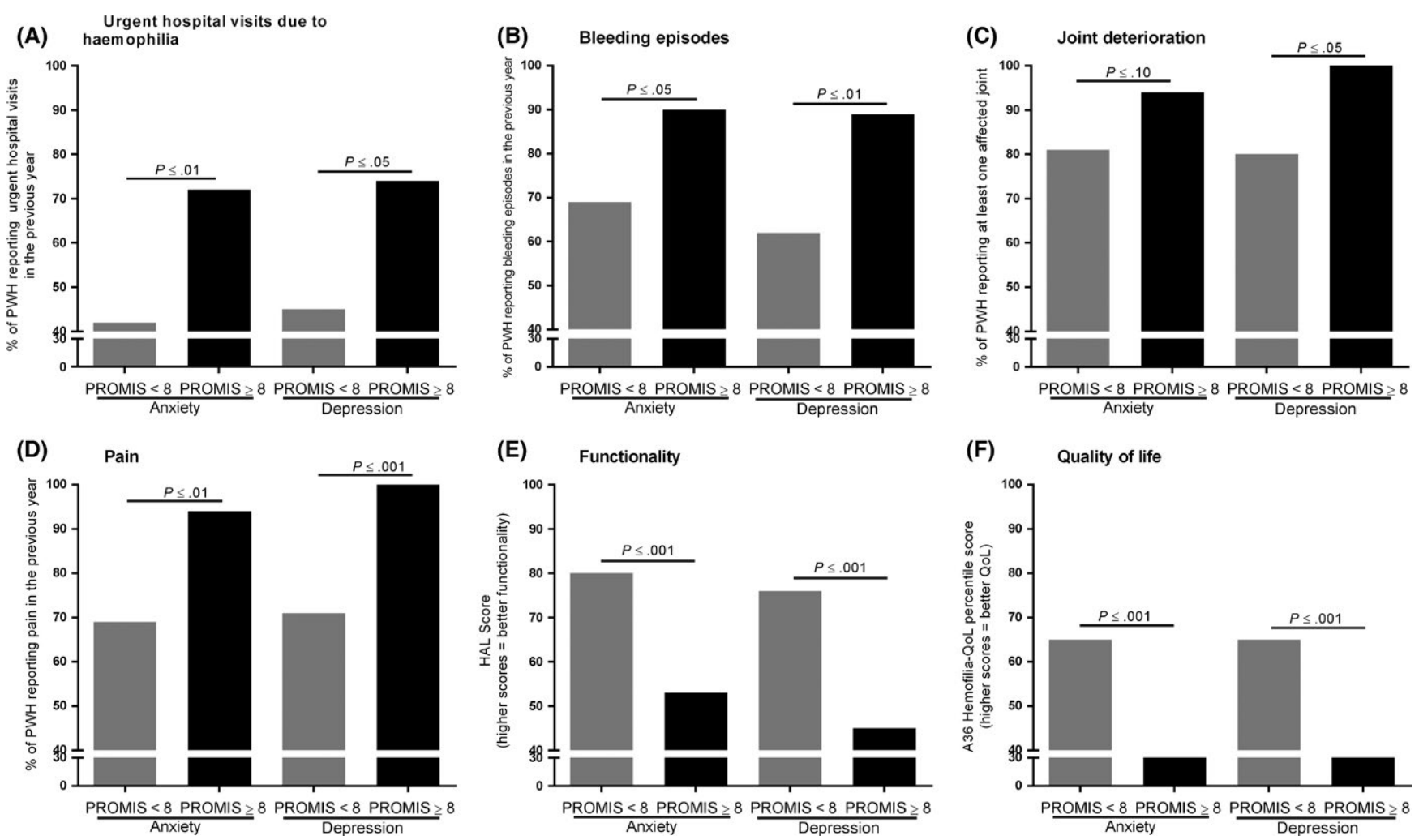

FIGURE 1 Differences among participants with and without significant anxiety/depression symptoms on haemophilia-related outcomes

(Table 1), who also perceived it as more intense (anxiety: $P<.001$, $r=.423$; depression: $P<.001, r=.476$ ) and as having more interference in their lives (anxiety: $P<.001, r=.472$; depression: $P<.001$, $r=.457$ ). Table 1 also indicates that a perception of symptoms unpredictability ("timeline cyclical") was more prominent among PWH presenting anxious $(P=.001, r=.351)$ and depressive $(P<.001$, $r=.380$ ) symptoms, who also had more negative haemophiliarelated consequences (anxiety: $P<.001, r=.479$; depression: $P<.001, r=.588$ ) and more negative emotional representations of the disease (anxiety: $P<.001, r=.370$; depression: $P<.001, r=.41$ ). Finally, both symptomatic groups reported worst levels of functionality (anxiety: $P<.001, r=.443$; depression: $P<.001, r=.443$ ) and quality of life (anxiety: $P<.001, r=.503$; depression: $P<.001$, $r=.408)$.

\section{3 | Factors associated with the presence of anxiety and depressive symptoms}

The results of the multivariate hierarchical logistic regression analyses are presented on Table 2. Model 1 shows that those being unemployed, retired or on medical leave were more likely to report both anxiety $(\mathrm{OR}=4.646 ; P=.004)$ and depressive symptoms $(\mathrm{OR}=3.333 ; \mathrm{P}=.029)$. Model 2 reveals that $\mathrm{PWH}$ who practice any type of physical activity tend to report less depressive symptoms than those who are sedentary $(O R=0.302 ; P=.024)$. Regarding pain, Model 3 uncovers that increased levels of pain interference were significantly associated with the presence of anxiety $(O R=1.397$;
$P=.011)$ and depressive $(O R=1.347 ; P=.037)$ symptoms among PWH. Focusing on illness perceptions, Model 4 evidences that a heightened perception of detrimental consequences related with haemophilia increases the likelihood of depressive symptomatology $(O R=1.600 ; P=.012)$. None of the IPQ-R subscales was associated with anxiety symptoms in the final multivariate model, despite their significant role in the previous univariate analysis.

\section{4 | DISCUSSION}

This study aimed to identify modifiable factors associated with anxiety and depression among $\mathrm{PWH}$. The findings highlighted a considerable proportion of participants with significant symptoms, and the association of anxiety and depression with clinical and psychosocial outcomes. It was also revealed that professional status, physical activity, pain interference and perception of haemophiliarelated negative consequences were associated with increased symptomatology. This was, to the best of our knowledge, the first study with PWH specifically aiming to identify factors associated to anxiety and depression that can be targeted by psychological interventions. In addition, the use of standardized scales for anxiety and depression screening was also a distinguishable feature of this investigation. Most studies in haemophilia field report anxiety/depression levels based on mental health subscales of health-related quality of life questionnaires, ${ }^{29-32}$ on self-reports of presence of anxiety/depression, ${ }^{9,33}$ or on unstandardized questions developed 
TA B LE 1 Sample characteristics and group comparisons between emotional distress groups (anxiety and depression) on sociodemographic, clinical and psychosocial measures

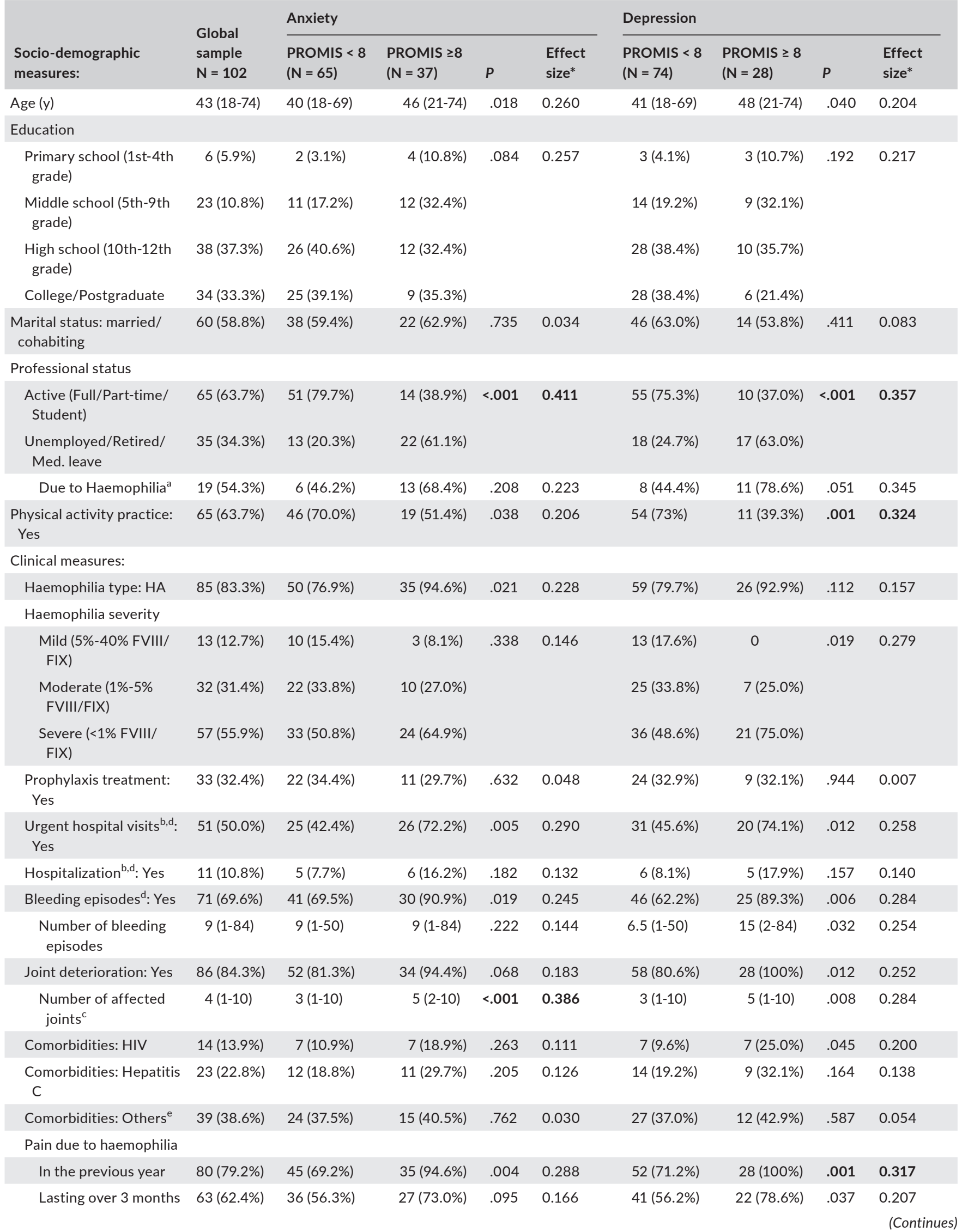


TABLE 1 (Continued)

\begin{tabular}{|c|c|c|c|c|c|c|c|c|c|}
\hline $\begin{array}{l}\text { Socio-demographic } \\
\text { measures: }\end{array}$ & $\begin{array}{l}\text { Global } \\
\text { sample } \\
\mathrm{N}=102\end{array}$ & \multicolumn{4}{|l|}{ Anxiety } & \multicolumn{4}{|l|}{ Depression } \\
\hline $\begin{array}{l}\text { More than once a } \\
\text { week }\end{array}$ & $41(40.6 \%)$ & $20(30.8 \%)$ & $21(56.8 \%)$ & .012 & 0.250 & $24(32.9 \%)$ & $17(60.7 \%)$ & .011 & 0.254 \\
\hline $\begin{array}{l}\text { Pain duration } \\
\text { (months) }\end{array}$ & $96(1-612)$ & $96(1-528)$ & $96(1-612)$ & .788 & 0.034 & $96(1-612)$ & $1(1-420)$ & .915 & 0.013 \\
\hline Pain interference ${ }^{d}$ & $3.3(0-9.9)$ & $1.6(0-8.4)$ & $5.3(0-9.9)$ & $<.001$ & 0.472 & $1.7(0-8.4)$ & $5.57(0-9.9)$ & $<.001$ & 0.457 \\
\hline \multicolumn{10}{|l|}{ Psychosocial measures: } \\
\hline PROMIS: Anxiety & $7(4-19)$ & $5(4-7)$ & $10(8-19)$ & - & - & $5(4-12)$ & $10(5-19)$ & $<.001$ & 0.640 \\
\hline PROMIS: Depression & $4(4-20)$ & $4(4-10)$ & $9(4-20)$ & $<.001$ & 0.643 & $4(4-7)$ & $10(8-20)$ & - & - \\
\hline IPQ-R: Consequences & $9(3-15)$ & $7(3-15)$ & $11(3-15)$ & $<.001$ & 0.479 & $8(3-15)$ & $12(7-15)$ & $<.001$ & 0.588 \\
\hline IPQ-R: Personal control & $8(3-15)$ & $7.3(3-11)$ & $8(3-15)$ & .232 & 0.118 & $8(3-11)$ & $8(3-15)$ & .734 & 0.034 \\
\hline $\begin{array}{l}\text { IPQ-R: Treatment } \\
\text { control }\end{array}$ & $7(3-12)$ & $6(3-12)$ & $7(3-12)$ & .401 & 0.083 & $6(3-12)$ & $7(3-12)$ & .232 & 0.118 \\
\hline $\begin{array}{l}\text { IPQ-R: Illness } \\
\text { coherence }\end{array}$ & $5.5(3-15)$ & $5(3-15)$ & $6(3-14)$ & .146 & 0.144 & $5(3-15)$ & $6(3-14)$ & .031 & 0.214 \\
\hline $\begin{array}{l}\text { IPQ-R: Emotional } \\
\text { representation }\end{array}$ & $6(3-15)$ & $5(3-15)$ & $7(3-15)$ & $<.001$ & 0.370 & $5(3-15)$ & $9(3-15)$ & $<.001$ & 0.410 \\
\hline HAL: Functionality & $\begin{array}{l}67.14 \\
(5-100)\end{array}$ & $\begin{array}{l}80.95 \\
(27-100)\end{array}$ & $53.33(5-100)$ & $<.001$ & 0.443 & $\begin{array}{l}76.79 \\
(22-100)\end{array}$ & $45.48(5-88)$ & $<.001$ & 0.443 \\
\hline
\end{tabular}

Continuous variables are presented as median (range) and categorical variables are presented as n (\%); A36 Hemofilia-QoL scores are presented as percentile; Bold=variables showing statistical significance at $p \leq 0.001$ and at least a medium effect size ( $>0.30)$.

PROMIS-Patient-Reported Outcomes Measurement Information System; IPQ-R-Illness Perception Questionnaire-Revised; HAL-Haemophilia Activities List.

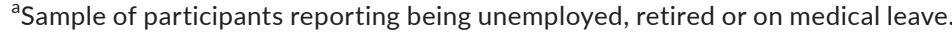

${ }^{b}$ Events that occurred due to haemophilia.

${ }^{\mathrm{c}}$ Number of joints pointed to be affected by haemophilia.

${ }^{\mathrm{d}}$ Data reporting to the previous year.

${ }^{e}$ Most commonly reported comorbidities were Hypertension ( $\left.N=17\right)$, Dyslipidaemias $(N=12)$ and Diabetes Type $2(N=9)$.

${ }^{*} r$ score for continuous variables and Pearson's phi $(\Phi)$ coefficient for nominal variables. To interpret the meaning of the $r$ score, Cohen's guidelines ${ }^{27}$ were used: a score above 0.50 is usually considered a large effect, between 0.50 and 0.30 a medium effect and between 0.30 and 0.10 a small effect size. The interpretation of Pearson's phi ( $\Phi)$ coefficient is analogous to the $r$ score, expressing the strength of association between two variables.

for the purpose of specific studies. ${ }^{34-36}$ The results of this investigation advance the knowledge on factors associated with anxiety and depression among $\mathrm{PWH}$, drawing relevant conclusions to inform interventions aiming to promote well-being among these patients.

\section{1 | Professional status}

The impact of haemophilia on professional activity has been widely demonstrated, with PWH reporting higher rates of unemployment and occupational disability than healthy controls. ${ }^{37,38}$ In this study, the association between professional status and both anxiety and depression remained significant even after controlling for clinical variables (disease severity and number of affected joints), showing that unoccupied patients (unemployed, retired or on medical leave) present higher symptomatology. These findings add to previous conclusions regarding $\mathrm{PWH}^{7,8}$ providing a research direction on the impact of haemophilia on emotional well-being and underscoring the relevance of a biopsychosocial model of haemophilia care that also considers professional status as a potential risk factor for poor mental health. Although further research is warranted, it can be suggested that PWH would benefit from more flexible employment policies. These could account for work absences due to haemophiliarelated complications, such as spontaneous bleeds, pain, mobility impairment or hospitalization. 
TAB LE 2 Multivariate hierarchical logistic regression analyses for factors associated with the presence of anxiety and depressive symptoms among PWH

\begin{tabular}{|c|c|c|c|c|c|c|}
\hline Models (Final Models) & \multicolumn{3}{|c|}{ PROMIS $\geq 8$-anxiety symptoms } & \multicolumn{3}{|c|}{ PROMIS $\geq 8$-depression symptoms } \\
\hline \multicolumn{7}{|c|}{ Model 1 (Professional status) } \\
\hline Age & 0.110 & $0.993(0.953-1.035)$ & .740 & 0.300 & $1.012(0.969-1.057)$ & .584 \\
\hline Education & 1.774 & $0.791(0.561-1.117)$ & .183 & 1.429 & $0.810(0.574-1.144)$ & .232 \\
\hline Professional status ${ }^{a}$ & 8.282 & $4.646(1.632-13.226)$ & .004 & 4.773 & $3.333(1.132-9.817)$ & .029 \\
\hline \multicolumn{7}{|l|}{ Model 2 (Physical activity) } \\
\hline Age & 0.100 & $1.006(0.967-1.048)$ & .752 & 0.254 & $1.012(0.967-1.059)$ & .614 \\
\hline Education & 3.509 & $0.734(0.531-1.014)$ & .061 & 2.421 & $0.758(0.534-1.075)$ & .120 \\
\hline \multicolumn{7}{|l|}{ Model 3 (Pain) } \\
\hline Age & 0.142 & $1.009(0.965-1.054)$ & .706 & 0.945 & $1.024(0.976-1.075)$ & .331 \\
\hline Education & 2.986 & $0.726(0.505-1.044)$ & .084 & 2.047 & $0.753(0.511-1.111)$ & .152 \\
\hline Haemophilia severity & 0.131 & $0.841(0.330-2.145)$ & .717 & 2.769 & $2.459(0.852-7.093)$ & .096 \\
\hline No of affected joints & 2.179 & $1.198(0.942-1.523)$ & .140 & 0.236 & $1.058(0.843-1.329)$ & .627 \\
\hline Pain intensity ${ }^{c}$ & 1.568 & $1.177(0.912-1.519)$ & .211 & 3.232 & $1.293(0.977-1.710)$ & .072 \\
\hline Pain interference ${ }^{c}$ & 6.536 & $1.397(1.081-1.806)$ & .011 & 4.336 & $1.347(1.018-1.783)$ & .037 \\
\hline \multicolumn{7}{|c|}{ Model 4 (IIlness perceptions) } \\
\hline Age & 0.169 & 1.009 (0.967-1.053) & 681 & 0.599 & $1.021(0.969-1.075)$ & .439 \\
\hline $\begin{array}{l}\text { Emotional } \\
\text { representation }\end{array}$ & 0.720 & $1.095(0.887-1.352)$ & .396 & 0.894 & $1.113(0.880-1.408)$ & .373 \\
\hline
\end{tabular}

MODEL 1: Anxiety: $\chi_{(5)}^{2}=28.282 P<.001$, Nagelkerke $R^{2}=0.342$, Final model correctly predicted $74.7 \%$ of all participants; Depression: $\chi_{(5)}^{2}=24.005$ $P<.001$, Nagelkerke $R^{2}=0.312$, Final model correctly predicted $80.8 \%$ of all participants.

Model 2: Anxiety: $\chi_{(5)}^{2}=21.833 P=.001$, Nagelkerke $R^{2}=0.269$, Final model correctly predicted $74.0 \%$ of all participants; Depression: $\chi_{(5)}^{2}=26.462$ $P<.001$, Nagelkerke $R^{2}=0.335$, Final model correctly predicted $78.0 \%$ of all participants.

Model 3: Anxiety: $\chi_{(6)}^{2}=39.982 P<.001$, Nagelkerke $R^{2}=0.452$, Final model correctly predicted $78.0 \%$ of all participants; Depression: $\chi_{(6)}^{2}=39.346$ $P<.001$, Nagelkerke $R^{2}=0.468$, Final model correctly predicted $80.0 \%$ of all participants.

Model 4: ANXIETY: $\chi_{(7)}^{2}=33.237 P<.001$, Nagelkerke $R^{2}=0.388$, Final model correctly predicted $78.0 \%$ of all participants; DEPRESSION: $\chi^{2}(7)=45.195$ $P<.001$, Nagelkerke $R^{2}=0.524$, Final model correctly predicted $84.0 \%$ of all participants.

$\mathrm{Cl}$, Confidence Interval.

a'Dichotomic variable: Active (Full/Part-time/Student) vs Unemployed/Retired/Med. leave.

${ }^{b}$ Dichotomic variable: no physical exercise practice vs occasional or regular practice of physical exercise.

${ }^{\mathrm{c} C}$ Continuous variable measured through a 0-10 NRS (Numerical Rating Scale).

${ }^{\mathrm{d} C}$ Continuous variable, subscale of IPQ-R: Illness Perception Questionnaire.

Bold=significant variables at $p \leq .05$

\section{2 | Physical activity}

The beneficial effects of physical activity on physical indicators of PWH is well documented, namely in promoting better physical function and endurance, improving bone metabolism and joint range of motion, increasing muscle strength and factor activity. $^{39-42}$

On the other hand, few studies have examined the impact of physical activity on psychosocial dimensions, albeit social and emotional benefits of physical activity are frequently suggested for 
$\mathrm{PWH}^{1,43}$ This fact is supported by present results, since $\mathrm{PWH}$ practicing physical activity had lower depressive symptoms, in agreement with previous findings. ${ }^{44}$ Therefore, exploring the effects of physical activity figures as a promising area of research in terms of promoting psychological health among $\mathrm{PWH}$, similarly to what has been described in other chronic illness. ${ }^{45,46}$ Ultimately, the efficacy of specific interventions for promoting physical activity, such as cognitive-behavioural strategies, behavioural activation or motivation interviewing should be analysed, as well as its effects on psychological symptoms of PWH.

\section{3 | Pain}

Pain is a central issue in haemophilia care, either due to haemarthrosis or to chronic arthropathy, ${ }^{47}$ which has been associated with higher emotional distress. ${ }^{8,33}$ Congruently in this study, participants with significant anxiety/depression symptoms were more likely to report higher pain frequency, with heightened intensity and interference. However, pain interference was the only dimension retaining significance in the regression models, being associated with anxiety and depression even after controlling for demographic and clinical variables. These findings highlight the relevance of implementing effective pain control strategies and reinforce the recent calls to action on the need to develop specific treatment guidelines for haemophilia pain management. ${ }^{48}$ These also underline the important role of wellprepared multidisciplinary teams for haemophilia care, ${ }^{1}$ skilled to deliver adequate and coordinated (non)pharmacological interventions for optimum pain management. Based on current results, it can be hypothesized that interventions aiming to decrease the perception of pain interference would be particularly helpful in reducing psychological distress symptoms. In this scope, psychological interventions are effective in fostering adaptive pain coping strategies, namely through specific cognitive techniques, such as cognitive restructuring, aiming to decrease pain intensity and perception of interference. ${ }^{49}$ This corroborates previous findings among $\mathrm{PWH}$, wherein the association between pain cognitions and emotional well-being is emphasized, namely the effect of pain reinterpreting techniques in pain management. ${ }^{47,50,51}$ Other strategies, such as relaxation, hypnosis and imagery training have been related to improved pain reports and reduced analgesic intake in former studies conducted among $\mathrm{PWH} .{ }^{52,53}$ Surprisingly, and despite their reported success, there is a scarcity of recent investigations focusing on psychological interventions for pain in $\mathrm{PWH}$ and, consequently, an important knowledge-gap on the potential effectiveness of such interventions.

\section{4 | Illness perceptions}

In face of a chronic illness, people develop individual representations of that disease, which have been shown to influence several healthrelated outcomes. ${ }^{54,55}$ The results from this study corroborate these findings, showing that patients with anxiety and depression had more threatening illness representations. This translates into a higher perception of symptoms unpredictability ("duration cyclical") and of negative consequences associated with the disease ("consequences"), as well as more negative feelings in face of haemophilia ("emotional representation"). In the multivariate analysis, a significant association was yielded between a negative perception of haemophiliarelated consequences and depressive symptomatology, above and beyond clinical factors. This finding shows that depressed patients are likely to perceive haemophilia as having a more negative impact on their lives than those without significant depressive symptoms. In line with these findings, perception of consequences has indeed been consistently associated with psychological distress in a variety of diseases. ${ }^{56,57}$ In the haemophilia field, the relevance of patients' individual perceptions has also been emphasized, with the perceived impact of the disease having a strong effect on clinical and psychosocial outcomes. ${ }^{58}$

These conclusions have relevant implications for haemophilia care planning, since it seems that promoting more adaptive illness perceptions may be an important strategy to prevent or decrease psychological distress. Namely, this could be achieved by diminishing the perception of negative impact of the disease, for example through psychoeducation, cognitive therapy or third-wave psychological interventions.

Despite the vast literature examining illness perceptions, studies in haemophilia field have only focused on their association with treatment adherence, namely on their influence in the adherence to clotting factor therapy. ${ }^{59-61}$ IIIness perceptions are therefore emerging as important variables to consider in haemophilia, with the results from this study reinforcing the need to expand the focus of research in this field.

\section{5 | Limitations}

This is a cross-sectional study and, therefore, temporal relations among findings, namely cause-effect associations, should not be established. Moreover, the classification of participants according to significant anxiety and depression symptoms was based on a self-report measure and not on formal clinical assessment of symptoms. Also, the sample size of the groups (with or without anxiety/ depression) is unbalanced, preventing from the use of more accurate statistical procedures. Future research with larger sample sizes and longitudinal assessment of outcomes would help in establishing more definite conclusions. Finally, it would be of interest to compare anxious/depressed patients in terms of objectively measured outcomes, such as a clinical/radiological assessment of joint status.

\section{5 | CONCLUSION}

Socio-demographic (eg age, gender) and disease-related (eg severity) characteristics have been associated with increased psychological symptoms in chronic pain patients, thus emerging as potential risk factors for higher emotional distress. ${ }^{62}$ However, the identification of these characteristics is of limited value for clinical intervention, due to their unchangeable nature. By identifying a specific set of modifiable variables that were associated with anxiety and 
depression, this study provided an evidence base for the development of preventive risk-targeted interventions aimed to foster emotional well-being, which might even contribute to the improvement of disease-related outcomes among PWH.

Although more research is needed to clearly identify specific risk factors for increased distress in PWH, it is clear that patients could substantially benefit from a complete multidisciplinary assessment, including an emotional distress screening that could identify specific needs and inform the development of appropriate treatment plans. ${ }^{1,8}$ Given the specific challenges of haemophilia across the lifespan, it is important to acknowledge them in adulthood and older age, and build on that knowledge to develop and test the effectiveness of specific psychological interventions. ${ }^{63}$

In sum, this study sheds light on factors that could be targeted to promote well-being and adaptation among PWH. Psychological interventions are an effective strategy for this purpose, which effectiveness should be tested in the haemophilia field.

\section{ACKNOWLEDGEMENTS}

The authors thank the Portuguese Haemophilia Association for the help in contacting the participants for this study.

\section{DISCLOSURES}

This work was supported by a grant from the Novo Nordisk HERO Research Grant 2015. P. R. Pinto has a grant (SFRH/ BPD/103529/2014) from the Portuguese Foundation for Science and Technology. The authors stated that they had no interests which might be perceived as posing a conflict or bias.

\section{AUTHOR CONTRIBUTION}

P. R. Pinto, A. C. Paredes and A. Almeida developed the concept for this paper. P. R. Pinto, A. C. Paredes and P. Moreira conducted the statistical analysis and interpreted the data. M. Carvalho, M. Lopes and S. Fernandes contributed with clinical expertise. All authors substantially contributed to research design, drafting and critically revision of the paper. All authors approved the final version of the manuscript.

\section{ORCID}

P. R. Pinto (iD http://orcid.org/0000-0003-3428-5801

\section{REFERENCES}

1. Srivastava A, Brewer AK, Mauser-Bunschoten EP, et al. Guidelines for the management of hemophilia. Haemophilia. 2013;19:e1-e47.

2. Bolton-Maggs PH, Pasi KJ. Haemophilias A and B. Lancet. 2003;361:1801-1809.

3. Siboni SM, Mannucci PM, Gringeri A, et al. Health status and quality of life of elderly persons with severe hemophilia born before the advent of modern replacement therapy. J Thromb Haemost. 2009;7:780-786.
4. Barlow JH, Stapley J, Ellard DR. Living with haemophilia and von Willebrand's: a descriptive qualitative study. Patient Educ Couns. 2007;68:235-242.

5. Flood E, Pocoski J, Michaels LA, Bell JA, Valluri S, Sasane R. Illustrating the impact of mild/moderate and severe haemophilia on health-related quality of life: hypothesised conceptual models. Eur J Haematol. 2014;93:9-18.

6. Cassis F. Psychosocial Care for People With Haemophilia. Montreal, QC: World Federation of Hemophilia (WFH); 2007.

7. Iannone $M$, Pennick $L$, Tom A, et al. Prevalence of depression in adults with haemophilia. Haemophilia. 2012;18:868-874.

8. Barry V, Lynch ME, Tran DQ, et al. Distress in patients with bleeding disorders: a single institutional cross-sectional study. Haemophilia. 2015;21:e456-e464.

9. Tran DQ, Barry V, Antun A, Ribeiro M, Stein S, Kempton CL. Physician trust and depression influence adherence to factor replacement: a single-centre cross-sectional study. Haemophilia. 2017;23:98-104.

10. Cassis FR, Querol F, Forsyth A, lorio A. Psychosocial aspects of haemophilia: a systematic review of methodologies and findings. Haemophilia. 2012;18:e101-e114.

11. Carlson LE, Bultz BD. Efficacy and medical cost offset of psychosocial interventions in cancer care: making the case for economic analyses. Psychooncology. 2004;13:837-849; discussion 850-836.

12. Williams AC, Eccleston C, Morley S. Psychological therapies for the management of chronic pain (excluding headache) in adults. Cochrane Database Syst Rev 2012;11:Cd007407.

13. Swirsky-Sacchetti T, Margolis CG. The effects of a comprehensive self-hypnosis training program on the use of factor VIII in severe hemophilia. Int J Clin Exp Hypn. 1986;34:71-83.

14. LaBaw W. The use of hypnosis with hemophilia. Psychiatr Med. 1992;10:89-98.

15. World Federation of Haemophilia. Report on the Annual Global Survey 2016. 2017.

16. Pinto PR, Paredes AC, Pedras S, et al. Sociodemographic, clinical, and psychosocial characteristics of people with hemophilia in Portugal: findings from the first national survey. TH Open. 2018;02:e54-e67.

17. Cleeland CS, Ryan KM. Pain assessment: global use of the Brief Pain Inventory. Ann Acad Med Singapore. 1994;23:129-138.

18. Pilkonis PA, Choi SW, Reise SP, Stover AM, Riley WT, Cella D. Item banks for measuring emotional distress from the patient-reported outcomes measurement information system (PROMIS(R)): depression, anxiety, and anger. Assessment. 2011;18:263-283.

19. Kroenke K, Yu Z, Wu J, Kean J, Monahan PO. Operating characteristics of PROMIS four-item depression and anxiety scales in primary care patients with chronic pain. Pain Med. 2014;15:1892-1901.

20. Moss-Morris R, Weinman J, Petrie K, Horne R, Cameron L, Buick D. The revised illness perception questionnaire (IPQ-R). Psychol Health. 2002;17:1-16.

21. Pinto PR, Mclntyre T, Nogueira-Silva C, Almeida A, Araujo-Soares $V$. Risk factors for persistent postsurgical pain in women undergoing hysterectomy due to benign causes: a prospective predictive study. J Pain. 2012;13:1045-1057.

22. Little RJA. A test of missing completely at random for multivariate data with missing values. J Am Stat Assoc. 1988;83:1198-1202.

23. Graham JW. Missing data analysis: making it work in the real world. Annu Rev Psychol. 2009;60:549-576.

24. Remor E, Arranz P, Quintana M, et al. Psychometric field study of the new haemophilia quality of life questionnaire for adults: the 'Hemofilia-QoL'. Haemophilia. 2005;11:603-610.

25. van Genderen FR, Westers P, Heijnen L, et al. Measuring patients' perceptions on their functional abilities: validation of the Haemophilia Activities List. Haemophilia. 2006;12:36-46.

26. Cronbach LJ. Coefficient alpha and the internal structure of tests. Psychometrika. 1951;16:297-334. 
27. Cohen J. Statistical Power Analysis for the Behavioural Sciences. Hillsdale, Michigan: Lawrence Erlbaum Associates; 1988.

28. Tabachnick BG, Linda SF. Using Multivariate Statistics. Harlow, UK: Pearson Education Limited; 2007.

29. Miners $\mathrm{AH}$, Sabin $\mathrm{CA}$, Tolley $\mathrm{KH}$, Jenkinson $\mathrm{C}$, Kind $\mathrm{P}$, Lee $\mathrm{CA}$ Assessing health-related quality-of-life in individuals with haemophilia. Haemophilia. 1999;5:378-385.

30. Stieltjes N, Torchet MF, Misrahi L, et al. Epidemiological survey of haemophiliacs with inhibitors in France: orthopaedic status, quality of life and cost-the 'Statut Orthopedique des Patients Hemophiles' avec Inhibiteur study. Blood Coagul Fibrinolysis. 2009;20:4-11.

31. Gringeri A, Mantovani LG, Scalone L, Mannucci PM. Cost of care and quality of life for patients with hemophilia complicated by inhibitors: the COCIS Study Group. Blood. 2003;102:2358-2363.

32. Kodra Y, Cavazza M, Schieppati A, et al. The social burden and quality of life of patients with haemophilia in Italy. Blood Transfus. 2014;12:s567-s575.

33. Witkop M, Neff A, Buckner TW, et al. Self-reported prevalence, description and management of pain in adults with haemophilia: methods, demographics and results from the Pain, Functional Impairment, and Quality of life (P-FiQ) study. Haemophilia. 2017;23:556-565.

34. Barlow JH, Stapley J, Ellard DR, Gilchrist M. Information and selfmanagement needs of people living with bleeding disorders:I a survey. Haemophilia. 2007;13:264-270

35. Forsyth AL, Gregory M, Nugent D, et al. Haemophilia Experiences, Results and Opportunities (HERO) Study: survey methodology and population demographics. Haemophilia. 2014;20:44-51.

36. Buckner TW, Witkop M, Guelcher C, et al. Management of US men, women, and children with hemophilia and methods and demographics of the Bridging Hemophilia B Experiences, Results and Opportunities into Solutions (B-HERO-S) study. Eur J Haematol. 2017;98:5-17.

37. Plug I, Peters M, Mauser-Bunschoten EP, et al. Social participation of patients with hemophilia in the Netherlands. Blood. 2008;111:1811-1815.

38. Hartl HK, Reitter S, Eidher U, Ramschak H, Ay C, Pabinger I. The impact of severe haemophilia on the social status and quality of life among Austrian haemophiliacs. Haemophilia. 2008;14:703-708.

39. Harris S, Boggio LN. Exercise may decrease further destruction in the adult haemophilic joint. Haemophilia. 2006;12:237-240.

40. Groen WG, den Uijl IEM, van der Net J, Grobbee DE, de Groot PG, Fischer K. Protected by nature? Effects of strenuous physical exercise on FVIII activity in moderate and mild haemophilia A patients: a pilot study. Haemophilia. 2013;19:519-523.

41. Parhampour B, Torkaman G, Hoorfar H, Hedayati M, Ravanbod R. Effects of short-term resistance training and pulsed electromagnetic fields on bone metabolism and joint function in severe haemophilia A patients with osteoporosis: a randomized controlled trial. Clin Rehabil. 2014;28:440-450.

42. Kargarfard M, Dehghadani M, Ghias R. The Effect of Aquatic Exercise Therapy on Muscle Strength and Joint's Range of Motion in Hemophilia Patients. Int J Prev Med. 2013;4:50-56.

43. Von Mackensen S. Quality of life and sports activities in patients with haemophilia. Haemophilia. 2007;13:38-43.

44. Lampinen P, Heikkinen RL, Ruoppila I. Changes in intensity of physical exercise as predictors of depressive symptoms among older adults: an eight-year follow-up. Prev Med. 2000;30:371-380.

45. Greenwood SA, Lindup $\mathrm{H}$, Taylor $\mathrm{K}$, et al. Evaluation of a pragmatic exercise rehabilitation programme in chronic kidney disease. Nephrol Dial Transplant. 2012;27:126-134.

46. Blumenthal JA, Sherwood A, Babyak MA, et al. Exercise and pharmacological treatment of depressive symptoms in patients with coronary heart disease: results from the UPBEAT (Understanding the Prognostic Benefits of Exercise and Antidepressant Therapy) study. J Am Coll Cardiol. 2012;60:1053-1063.

47. Santavirta N, Bjorvell H, Solovieva S, Alaranta H, Hurskainen $\mathrm{K}$, Konttinen YT. Coping strategies, pain, and disability in patients with hemophilia and related disorders. Arthritis Rheum. 2001;45:48-55

48. Humphries TJ, Kessler CM. Pain in haemophilia: are we listening? Haemophilia. 2016;22:175-178.

49. Ehde DM, Jensen MP. Feasibility of a cognitive restructuring intervention for treatment of chronic pain in persons with disabilities. Rehabil Psychol. 2004;49:254-258.

50. Elander J, Robinson G, Mitchell K, Morris J. An assessment of the relative influence of pain coping, negative thoughts about pain, and pain acceptance on health-related quality of life among people with hemophilia. Pain. 2009;145:169-175.

51. Elander J, Morris J, Robinson G. Pain coping and acceptance as Iongitudinal predictors of health-related quality of life among people with haemophilia-related joint pain. Eur J Pain. 2013;17:929-938.

52. Varni JW, Gilbert A. Self-regulation of chronic arthritic pain and long-term analgesic dependence in a haemophiliac. Rheumatol Rehabil. 1982;21:171-174.

53. Varni JW. Self-regulation techniques in the management of chronic arthritic pain in hemophilia. Behav Ther. 1981;12:185-194.

54. Crawshaw J, Rimington H, Weinman J, Chilcot J. Illness perception profiles and their association with 10-year survival following cardiac valve replacement. Ann Behav Med. 2015;49:769-775.

55. Jaremo P, Arman M, Gerdle B, Larsson B, Gottberg K. Illness beliefs among patients with chronic widespread pain - associations with self-reported health status, anxiety and depressive symptoms and impact of pain. BMC Psychol. 2017;5:24.

56. Stafford L, Berk M, Jackson HJ. Are illness perceptions about coronary artery disease predictive of depression and quality of life outcomes? J Psychosom Res. 2009;66:211-220.

57. McCorry NK, Dempster M, Quinn J, et al. Illness perception clusters at diagnosis predict psychological distress among women with breast cancer at 6 months post diagnosis. Psychooncology. 2013;22:692-698.

58. Holstein K, von Mackensen S, Bokemeyer C, Langer F. The impact of social factors on outcomes in patients with bleeding disorders. Haemophilia. 2016;22:46-53.

59. Lamiani G, Strada I, Mancuso ME, Coppola A, Vegni E, Moja EA. Factors influencing illness representations and perceived adherence in haemophilic patients: a pilot study. Haemophilia. 2015;21:598-604.

60. van Os SB, Troop NA, Sullivan KR, Hart DP. Adherence to prophylaxis in adolescents and young adults with severe haemophilia: a quantitative study with patients. PLoS ONE. 2017;12:e0169880.

61. Llewellyn CD, Miners AH, Lee CA, Harrington C, Weinman J. The illness perceptions and treatment beliefs of individuals with severe haemophilia and their role in adherence to home treatment. Psychol Health. 2003;18:185-200.

62. Soucie JM, Grosse SD, Siddiqi AE, et al. The effects of joint disease, inhibitors and other complications on health-related quality of life among males with severe haemophilia A in the United States. Haemophilia. 2017;23:e287-e293.

63. Pinto PR, Paredes AC, Costa P, et al. Effectiveness of two psychological interventions for pain management, emotional regulation and promotion of quality of life among adult Portuguese men with haemophilia (PSY-HaEMOPEQ): study protocol for a single-centre prospective randomised controlled trial. BMJ Open. 2017;7:e016973.

How to cite this article: Pinto PR, Paredes AC, Moreira P, et al. Emotional distress in haemophilia: Factors associated with the presence of anxiety and depression symptoms among adults. Haemophilia. 2018;00:1-10.

https://doi.org/10.1111/hae.13548 